\title{
Principio de precaución en la acción de tutela. Casos ambientales colombianos
}

\section{Precaution principle in tutela action. Colombian environmental cases}

\author{
DOI: https://doi.org/10.17981/juridcuc.16.1.2020.08
}

Fecha de Recepción: 28/08/2019. Fecha de Aceptación: 17/12/2019

\author{
Andrea Carolina Ariza Sánchez \\ Universidad del Magdalena. Santa Marta (Colombia) \\ aariza@unimagdalena.edu.co
}

Para citar este artículo:

Ariza, A. (2020). Principio de precaución en la acción de tutela. Casos ambientales colombianos. JURIDICAS CUC, 16(1). 197-224. DOI: http://dx.doi.org/10.17981/juridcuc.16.1.2020.08

\section{Resumen}

El principio de precaución, pretende prevenir daños o afectaciones al medio ambiente, bien jurídico de máxima protección, debido a la vulneración a la que se encuentra sometido y su relación con la existencia de la vida humana. $\mathrm{Su}$ introducción a la normatividad nacional ha permitido otorgar herramientas de interpretación a los operadores judiciales al momento de desatar problemas jurídicos ambientales. Por ello, resulta importante analizar cómo ha sido su aplicación y desarrollo en la acción de tutela por parte de la Corte Constitucional. Para resolver esta problemática, se tomó la metodología del estudio de casos, en la que fueron seleccionados distintos fallos judiciales para poder determinar los postulados hermenéuticos de aplicación del principio de precaución, con el fin de establecer posibles soluciones a casos futuros y analizar su impacto dentro de la solución de estas problemáticas ambientales. De este análisis se concluye la importancia del abordaje del principio de precaución para robustecer los lineamientos jurisprudenciales permitiendo solucionar casos ambientales en distintas acciones constitucionales, en las que se demuestra el compromiso ambiental de Colombia frente a los compromisos internacionales. Este artículo representa un valor importante para los académicos, operadores judiciales, abogados y accionantes, al delimitar los distintos escenarios de aplicación del principio de precaución, resaltando los parámetros de interpretación y aportando a las bases argumentativas para sostener posturas en los casos ambientales.

Palabras clave: Acción de tutela; medio ambiente sano; principio de precaución

\begin{abstract}
The precautionary principle aims to prevent damage to or impairment of the environment, a legal good of maximum protection, due to the violation to which it is subject and its relationship with the existence of human life. Its introduction to national regulations has allowed the granting of interpretation tools to judicial operators at the moment of unleashing environmental legal problems. Therefore, it is important to analyze how it has been applied and developed in the action of protection by the Constitutional Court. To solve this problem, the methodology of the case study was taken, in which different judicial rulings were selected to be able to determine the hermeneutic postulates of the application of the precautionary principle, in order to establish possible solutions to future cases and analyze their impact within the solution of these environmental problems. From this analysis we conclude the importance of the approach of the precautionary principle to strengthen the jurisprudential guidelines allowing to solve environmental cases in different constitutional actions, in which the environmental commitment of Colombia in front of international commitments is demonstrated. This article represents an important value for academics, judicial operators, lawyers and plaintiffs, by delimiting the different scenarios of application of the precautionary principle, highlighting the parameters of interpretation and contributing to the argumentative bases to sustain positions in environmental cases.
\end{abstract}

Keywords: Guardianship action; healthy environment; precautionary principle 


\section{INTRODUCCIÓN}

El tema de este artículo de investigación se encuentra justificado, toda vez, que hoy dentro de los puntos más importantes de las agendas de los Estados, está el aspecto de la conservación del medio ambiente, como instrumento eficaz para conservar la salud del ser humano y de los recursos y ecosistemas, siendo posible la implementación de acciones legales que busquen su protección.

Para cumplir con los objetivos de esta investigación, se estudió la normatividad aplicable al principio de precaución y su evolución jurisprudencial, en acciones de tutela emitidas por la Corte Constitucional colombiana, en sede de revisión; con el fin de dar a conocer en la comunidad académica estos avances y su importancia para la prevención de los daños ambientales, evitando futuros perjuicios a la salud humana.

La idea de desarrollar este artículo surgió del siguiente problema jurídico: ¿Cuál ha sido la aplicación del principio de precaución vigente en Colombia a partir de las acciones de tutela resueltas por la Corte Constitucional?

Para resolver este problema, se utilizó el método del estudio del caso para descubrir la intervención del principio de precaución en los fallos de tutela, los cuales fueron tomados como muestra. Se analizaron las sentencias de tutela emitidas por la Corte Constitucional, para identificar los parámetros de caracterización del principio de precaución y sus causales de procedencia en este tipo de acción constitucional.

La hipótesis de esta investigación es como el empleo del principio de precaución en Colombia, de acuerdo a los tratados internacionales pertinentes, teniendo en cuenta la protección a la vida y salud del ser humano desde la defensa al medio ambiente, por hechos de los cuales no haya certeza que sean causantes de un daño, pero existan serios indicios que permiten afirmar que sí; evento en el cual, resulta procedente la acción de tutela.

Este artículo estuvo guiado por el objetivo general de analizar el desarrollo en materia normativa y jurisprudencial del principio de precaución; con los objetivos específicos de realizar una recopilación de la normatividad nacional sobre el principio de precaución y analizar 
el desarrollo jurisprudencial del principio de precaución en sede de revisión de las acciones de tutela, por parte de la Corte Constitucional.

\section{Principio de precaución. Definición, consagración nacional e internacional, funciones y requisitos de aplicación}

Con el fin de aproximarnos conceptualmente con el contenido de este artículo, resulta indispensable definir el principio de precaución, conforme a la doctrinal nacional e internacional

El argentino Roberto Andorno (2008) indica que "el principio de precaución orienta las decisiones, frente a la existencia de sospechas de riesgo a la salud pública o el medio ambiente, con el uso de ciertos productos o tecnologías, sin existir prueba científica del riesgo" (pp. 345-346).

Este mismo autor, después de dar una definición sobre el principio, indicó que está encaminado a orientar las políticas de protección del ambiente de posibles daños, sin interesar la certeza, sobre si alguna actividad repercute gravemente en el ambiente, explica el origen de este principio en la legislación alemana de 1971 (Andorno, 2008), y como a partir de allí se ha expandido en los diferentes tratados y legislaciones globales.

Paralelamente a lo anterior, el principio de precaución supera los límites de la ciencia, y protege al medio ambiente y la salud humana más allá de un nexo de causalidad entre dos situaciones. "Cuando una actividad o producto representa una amenaza potencial para el medio ambiente o la salud pública, deben tomarse las medidas pertinentes, aun cuando la relación causa-efecto no haya podido demostrarse científicamente de forma concluyente" (Andorno, 2008, p. 346).

Pastorino citado por Kamada (2012), recuerda que "Prieur dice que se trata de una nueva forma de prevención ante la ignorancia respecto de los efectos que pueden tener ciertas actividades o sustancias o técnicas" (p. 6). Prieur lo que propone es el avance de las ciencias humanas como el derecho sobre las ciencias exactas tal como lo es la bioquímica. El principio precautorio es manifestación del avance de las ramas sociales sobre las exactas. 
En dicho sentido, el avance de las ramas sociales traslada al principio de precaución en la pirámide de oposición a las actividades humanas irresponsables y carentes de previsión de una amenaza o daño a la vida, a pesar de que estas actividades humanas no tengan una etiqueta llamativa de "perjudicial", el mencionado principio no deja al azar sus efectos o consecuencias de dicha etiqueta cuando de proteger el medio ambiente y sus habitantes se trata. En razón de ello, como más adelante se vera, el principio de precaución ha sido reconocido e implementado en las normas internacionales y nacionales y con ello adaptado en las políticas públicas de los gobiernos. Previo a ello, es dable precisar:

El hecho de no conocer cuáles son las consecuencias a corto, mediano o largo plazo de las actividades humanas sobre el medio ambiente, no se configura en un pretexto suficiente como para evadir las responsabilidades por parte de la administración entorno a la implementación de políticas que busquen prevenir el deterioro del medio ambiente (Rodriguez y Vargas, 2017, p. 19).

En conclusión, el principio de precaución se encarga de proteger el medio ambiente, cuando exista una certeza mínima más no absoluta de la afectación que podría sufrir el entorno por una acción determinada. La no aplicación de este principio generaría, un desequilibrio violatorio a toda protección ambiental moderna.

Para los fines de nuestro argumento, es dable afirmar que, si el derecho se supedita a la exactitud absoluta de la ciencia, nunca se podría proteger al medio ambiente, lo cual, a pesar de poder producir daño, la ciencia no lo confirme. Por lo anterior, la razón de ser del principio es esa: proteger al medio ambiente de un posible daño, aunque no se tenga la seguridad absoluta de que cierta actividad lo afecte.

\section{Consagración Nacional e Internacional}

Previo a profundizar sobre el origen y evolución nacional e internacional del principio de precaución, es de resaltar que este deviene del raciocinio y conducta humana como todos los demás principios 
existentes y llevados al derecho positivo, y fundamentalmente se encamina a la necesidad de supervivencia, cuidando la vida y la salud frente a lo desconocido o inseguro. Es así como:

El principio de precaución tiene su origen en un antiguo canon del comportamiento humano, correspondiéndose a una visión renovada de la ancestral concepción de "prudencia" ante lo incierto, lo desconocido. En la "prudencia" se enlazan la "conjetura" basada en la "memoria", la "inteligencia" con su razonamiento inductivodeductivo (analogía con lo conocido) y la "providencia" (disposición anticipada para evitar o minimizar los daños o males supuestos y temidos). Implica una actitud de reserva, circunspección, previsión (Drnas, 2009).

Del análisis de la Sentencia C-293 (2002), se desglosa un poco la historia de este principio. Se “... pone de presente que el principio de precaución se formuló en Alemania en los años 70, con el fin de asegurar el resarcimiento al menoscabo de la vida humana, originado en los efectos nocivos de los productos químicos" (p. 1). Así mismo, el autor (Cafferatta, 2004) indica que “... la primera expresión del principio de precaución surgió en los años 1970 con Vorsorgeprinzip en el campo del derecho alemán del ambiente"; con el fin de tomar medidas contra los distintos fenómenos de contaminación ambiental (De Cozár, 2005).

Sobre este mimo aspecto, Agudelo y López (2009) citan el derecho alemán Vorsongende Umweltpolitik del Gobierno Federal Alemán de 1976 donde se consagró el principio de precaución así: "La política ambiental no se agota en la defensa contra peligros amenazantes y la reparación de daños ya acaecidos. Una política ambiental precautoria (vorsongende Umweltpolitik) exige, más allá de eso, que los fundamentos de la naturaleza sean apropiadamente valorados y conservados" (p. 17).

Con posterioridad, en junio de 1992 se expide la Declaración de Río de Janeiro sobre Medio Ambiente y Desarrollo Sostenible (Organización de las Naciones Unidas-ONU, 1992), y el quinceavo principio de esta declaración dicta: 
Principio 15. Con el fin de proteger el medio ambiente, los Estados deberán aplicar ampliamente el criterio de precaución conforme a sus capacidades. Cuando haya peligro de daño grave o irreversible, la falta de certeza científica absoluta no deberá utilizarse como razón para postergar la adopción de medidas eficaces en función de los costos para impedir la degradación del medio ambiente (ONU, 1992)

Consecuencialmente con lo anterior, al estar internacionalmente consagrado el principio de precaución, en el mismo año del tratado, para el 22 de diciembre en Colombia se expide la Ley 99, en la que se consagró este principio. Esta ley es "por la cual se crea el Ministerio del Medio Ambiente, se reordena el Sector Público encargado de la gestión y conservación del medio ambiente y los recursos naturales renovables, (...) y se dictan otras disposiciones" (Ley 99, 1992).

Conviene señalar que al ser el principio de precaución, el orientador de políticas para proteger el medio ambiente aun sin tener la certeza de que una actividad ataca el medio o no, pues debía estar presente en las funciones del Ministerio del Medio Ambiente y por ello el artículo $1^{\circ}$ numeral 6 de esta ley ilustra:

La formulación de las políticas ambientales tendrá en cuenta el resultado del proceso de investigación científica. No obstante, las autoridades ambientales y los particulares darán aplicación al principio de precaución conforme al cual, cuando exista peligro de daño grave e irreversible, la falta de certeza científica absoluta no deberá utilizarse como razón para postergar la adopción de medidas eficaces para impedir la degradación del medio ambiente (Ley 99, art. 1, 1992).

Posteriormente, el 9 de mayo de 1992 en la ciudad de Nueva York fue adoptada la Convención Marco de las Naciones Unidas sobre el Cambio Climático y el 21 de marzo de 1994 esta entra en vigor (United Nations Framework Convention on Climate ChangeUNFCCC, 1992). Con la Ley 164 (1994) el país aprueba dicha convención y la incorpora al ordenamiento, incluyendo nuevamente el principio de precaución en el artículo $3^{\circ}$ : 
Las Partes deberían tomar medidas de precaución para prever, prevenir o reducir al mínimo las causas del cambio climático y mitigar sus efectos adversos. Cuando haya amenaza de daño grave o irreversible, no debería utilizarse la falta de total certidumbre científica como razón para posponer tales medidas, tomando en cuenta que las políticas y medidas para hacer frente al cambio climático deberían ser eficaces en función de los costos a fin de asegurar beneficios mundiales al menor costo posible. A tal fin, esas políticas y medidas deberían tener en cuenta los distintos contextos socioeconómicos, ser integrales, incluir todas las fuentes, sumideros y depósitos pertinentes de gases de efecto invernadero y abarcar todos los sectores económicos. Los esfuerzos para hacer frente al cambio climático pueden llevarse a cabo en cooperación entre las Partes interesadas (Ley 164, art. 3, 1994).

Y por último, con la Ley 1523 (2012): "Por la cual se adopta la política nacional de gestión del riesgo de desastres, se establece el Sistema Nacional de Gestión del Riesgo de Desastres y se dictan otras disposiciones", toma protagonismo nuevamente el principio de precaución; y por primera vez en el ordenamiento legal se añade expresamente éste en el artículo $3^{\circ}$ numeral 8 en estos términos:

8. Principio de precaución: Cuando exista la posibilidad de daños graves o irreversibles a las vidas, a los bienes y derechos de las personas, a las instituciones y a los ecosistemas como resultado de la materialización del riesgo en desastre, las autoridades y los particulares aplicarán el principio de precaución en virtud del cual la falta de certeza científica absoluta no será óbice para adoptar medidas encaminadas a prevenir, mitigar la situación de riesgo (Ley 1523, art. 3, 2012)

Bajo esta misma secuencia, se pueden observar otros tratados internacionales, y convenios como por ejemplo los Tratados de Maastricht (1992) y Ámsterdam (1997), de la unión europea, además de la declaración de Bergen sobre Desarrollo Sostenible (1990), la Convención sobre la Diversidad Biológica (1992) y el Protocolo de Cartagena de Bioseguridad (2000) (citados por Andorno, 2008, p. 346). De esta forma, se aprecia con claridad la inmersión del principio de precaución en un escenario jurídico nacional e internacional con fundamentos sólidos y parámetros de razonabilidad. 
Resumiendo, se puede afirmar la existencia de una consagración del principio de precaución o precautorio en diversas legislaciones estatales. La necesidad de todos los Estados consistente en proteger el medio ambiente para su bienestar futuro, y el bienestar global de la especie humana, ha permitido sabiamente la creación e implementación efectiva de medidas precautorias.

A partir del desarrollo internacional del principio, el Estado colombiano ha fijado las prerrogativas nacionales internas, y por ello ha vinculado a su ordenamiento jurídico algunos tratados y convenios cosmopolitas con el objetivo tanto de proteger el medio ambiente, tanto de tener un estatuto biológico fuerte.

\section{Funciones}

Descendiendo en el estudio, se tiene que la función principal, del principio de precaución es la de orientar las políticas ambientales de un Estado a la protección del medio ambiente, si bien, no se tiene certeza bajo una relación de causa-efecto de la afectación directa de una actividad al medio ambiente.

De igual modo, de la función indicada se deriva que el principio en estudio es un instrumento de gran importancia tanto en las políticas de amparo del medio ambiente, como en la apropiación que del mismo realicen los jueces en sus providencias, teniendo presente que su implementación no debe exigir una garantía de evidencia o prueba absoluta de un daño ante el apremio de salvaguardar un derecho. La utilización de distintas acciones judiciales constituyen la materialización de la democracia como resultado de los avances sociales y culturales (Pabón, 2018), así mismo, la democracia está en permanente construcción y supone la formación de la ciudadanía (Reales, 2016).

El principio está encaminado a hacer énfasis en la imposibilidad de usar la ausencia de seguridad científica, como excusa para tomar medidas sobre posibles daños graves e irreversibles. No implica la arbitrariedad de la autoridad al imponer sanciones, tan solo afirma que la ausencia de certeza no le impide tomar medidas a favor del ambiente. 
El desarrollo del principio de precaución, como se analizará de manera jurisprudencial, no solo tiene consecuencias encaminadas en prevenir el daño, sino que implica la consecución de una responsabilidad para la autoridad y para el resto de la comunidad quienes deberán ejercer acciones públicas y privadas para dirigir su actuar en actividades que no afecten el medio ambiente y en consecuencia la vida y la salud de la población.

De lo que se deriva que la aplicación de la realidad a estos casos, constituye una configuración de la investigación acción en la cual se busca llevar a la realidad la solución de las situaciones fácticas planteadas y generar dinámicas investigativas (Bechara, 2018).

Sobre esta arista, se busca la aproximación de la incertidumbre científica, sobre informaciones que resultan indispensables para la toma de decisiones políticas y así prevenir el daño; evitando con ello equivocaciones (Sánchez, 2004).

De igual manera, ha sido utilizado como criterio hermenéutico para solucionar controversias jurídicas. En efecto, cuando un servidor público no tiene la convicción absoluta de la afectación o vulneración del derecho de una persona por determinada acción, pero tiene un gran indicio sobre ello, pues deberá interpretar la situación mediante el principio precautorio y proteger los posibles derechos afectados, tal como lo planteó la Corte Constitucional en la Sentencia C-293 (2002).

\section{Requisitos de aplicación}

Resulta necesario advertir, que la Ley 99 (1992) fue demandada por inconstitucionalidad, debate resuelto mediante Sentencia C-293 (2002) de la Corte Constitucional. En dicho debate se resolvió la exequibilidad del principio de precaución, haciendo énfasis en los requisitos para su aplicación: 1) la existencia de un peligro de daño; 2) que este sea grave e irreversible; 3) que existe un principio de certeza científica; 4) que la decisión adoptada por la autoridad esté encaminada a impedir la degradación del medio ambiente y 5) la motivación en la decisión (Sentencia C-293, 2002). 
Aunado a lo anterior, ese pensamiento ecológico y conservista del medio ambiente, fue ratificado en la Sentencia C-671 (2001), la cual señala la necesidad de las normas ecologistas y de las políticas protectoras, aún más en un país biológicamente diverso como Colombia. Podemos decir que las causas de la degradación ambiental son en su mayoría antropogénicas, es decir, producto de la actividad humana. Por consiguiente, la protección del medio ambiente dentro del derecho internacional se ha vuelto prioridad. Estas actividades encaminadas a suplir necesidades humanas se incrementaron por los procesos industriales del siglo pasado, generando polución, calentamiento global y daños grandes a los ecosistemas. Aunque el derecho internacional le brinde autonomía a los Estados, al ser la situación ambiental común a todos estos, las acciones hechas por cada uno de ellos son vitales para la supervivencia futura de todos, por ello herramientas como el principio precautorio están globalizadas y son necesitadas por la raza humana en su conjunto (Sentencia C-671, 2001).

De allí, surgen las razones que sustentan la exequibilidad del principio de precaución, nacida de los compromisos que devienen de los convenios y acuerdos internacionales ratificados por Colombia. De igual manera, si un pacto internacional no está ratificado por el país, su existencia muestra un indicio sobre su utilidad a la hora de proteger el medio ambiente, lo cual se suple en Colombia con las leyes dispuestas para ello, como se ha mencionado.

Bajo los anteriores parámetros, el principio de precaución se aplica cuando el riesgo o la magnitud del daño producido no son relevados con anticipación, toda vez, que existe desconocimiento, los efectos de una acción (Sentencia C-703, 2010). Lo cual generalmente ocurre, porque no existe conocimiento científico cierto referente a las consecuencias de situaciones o actividades, a pesar de conocer lo nocivo de los efectos, y además tomando en cuenta los 5 requisitos enumerados con anterioridad.

Aunado a los citados requisitos, en la doctrina jurídica como lo expone Artigas (citado por Rodriguez \& Vargas, 2017), también se evidencian elementos que hacen parte de principio en estudio como son: 
- Anticipación preventiva: se refiere concretamente a la toma de decisiones anticipadas, sin llegar a la necesidad de esperar a las pruebas de carácter científico, toda vez que si no se puede hacer, esto causaría un mayor costo para la sociedad y el medio ambiente.

- Salvaguardia: es necesario proteger al medio ambiente de manera primordial, para ello es un deber la abstención de aprovechamiento de recursos indeseables para ellos (Granja, 2010, p. 120)

De lo expuesto se deriva que, el principio de precaución no es utilizado arbitrariamente. La posibilidad de actuar ante la ausencia de certeza absoluta no implica per se la arbitrariedad en su uso al no estar seguros sobre la afectación al ambiente. Es por ello, que la existencia de prerrogativas que aritméticamente deben estar presentes con la intención de tomar este fundamento, so pena de no ser viable su configuración. Se puede decir que en Colombia esos parámetros están asentados claramente en la jurisprudencia de la Corte Constitucional, situación que genera una amplia seguridad jurídica.

\section{Acciones de tutela y el principio de precaución}

En el artículo 86 de la Constitución Política colombiana (1991), reglamentada por el Decreto 2591 (1991), se contempla la Acción de Tutela, mecanismo judicial idóneo para solicitar el amparo de los derechos fundamentales. En la labor de hermenéutica nuestra honorable Corte Constitucional ha emprendido una interpretación y aplicación del principio de precaución, cuando se observe la afectación de los derechos de salud, salubridad pública y vida, aún si no se tiene certeza que alguna acción genere afectación a la vida humana.

Es así como la jurisprudencia constitucional colombiana ha estudiado, valorado, evaluado y aplicado este principio de precaución en diversos casos que exigen de esta honorable corporación la salvaguarda de derechos fundamentales y su posición activa y concreta sobre la prevalencia de los derechos fundamentales ante los riesgos que alguna actividad humana genere, siempre bajo la observancia de no descartar una decisión drástica en el amparo de los derechos frente a los distintos riesgos existentes bajo la regla de la precaución 
extrema. "En ejercicio de la regla de precaución extrema, la Corte ha exigido la cesación de actividades que pueden generar riesgos, como la colocación de antenas de telefonía móvil cerca a lugares donde residen niños, ancianos o personas enfermas" (Corte Constitucional, Sentencia T-236, 2017).

Finalmente, antes de plantear los casos analizados en el plano jurisprudencial, resulta menester resaltar un aparte considerativo de la Corte Constitucional en una de sus sentencias sobre el principio en estudio, en razón a que dicha consideración enaltece la actitud activa para prever situaciones que pongan en riesgo un derecho fundamental como el del medio ambiente.

Conforme a ello, el principio de precaución no sólo se ejercita conforme a las consecuencias de los actos, sino que busca tomar una posición de adelantarse, para prevenir un próximo acontecimiento medioambiental y de esta manera mejorar o proteger la naturaleza (Corte Constitucional. Sentencia C-595, 2010).

Se debe precisar como a través de la Sentencia T-021 (2019), la Corte Constitucional relaciona el principio de precaución como uno de los elementos indispensables dentro de la justicia ambiental, conforme a la constitucionalización del derecho ambiental, ligado a los tratados internacionales, razones suficientes para dejar con plena validez actos administrativos de las autoridades ambientales, frente a la restricción de ciertas actividades que realizan las comunidades y que impactan los biomas. Sin embargo, esta sentencia, no se tomara como análisis de caso, toda vez que no guarda un hilo conductor con las sentencias seleccionadas para revisión en esta disertación.

\section{Primer caso (Sentencia T-1062 de 2001)}

Inicia esta acción de tutela en marzo de 2010, Lucila Baena Parra solicitó la tutela de sus derechos, que consideró vulnerados por la administración de su conjunto residencial, quien celebró un contrato de arrendamiento con COCELCO S.A, la otra entidad tutelada, siendo el objeto de este contrato el arriendo de zonas comunes del conjunto, con miras a colocar entenas de telecomunicaciones en él. 
La parte actora, solicita el amparo transitorio de los derechos a la integridad física y personal, núcleo familiar, a la vida y medio ambiente, toda vez, que Lucila Baena Parra se encuentra en un estado de salud delicado, y al vivir en el último piso del edificio donde habían colocado 18 antenas de telefonía celular en el techo y una antena tipo plato para microondas, debido al contrato de arrendamiento entre el conjunto y la empresa de telecomunicaciones, se le empeoró dicho estado debido muy posiblemente a las radiaciones electromagnéticas proferidas por los aparatos.

Los actores afirmaron, que al iniciar las actividades de las antenas ubicadas en la estación base, el estado de salud de la señora Baena se afectó negativamente, con necesidad de cambiar de residencia. La primera instancia de la tutela, ilustra por qué es útil el principio de precaución en el ordenamiento jurídico colombiano. El a quo niega la protección de los derechos a la vida e integridad física y personal y la protección a su núcleo familiar; toda vez que, considera que al no ser serios ni científicos, los estudios sobre los efectos en la salud humana, de las ondas de telefonía móvil y otras ondas receptoras, pasan a ser especulativos (Sentencia T-1062, 2001). Razón por la cual niega la acción de tutela.

De similar forma respondió el Ad quem al afirmar en una de las partes de su sentencia lo siguiente:

Es que conforme con el dictamen pericial allegado, el funcionamiento de los equipos electromagnéticos y de las antenas, de la empresa Celumóvil en el edificio Portón de San Carlos, no están contaminando el medio ambiente en él, ya que los niveles de exposición a los moradores del mismo, están muy por debajo de los niveles generalmente considerados por los expertos como nocivos; y como consecuencia natural, no puede afirmarse, como un hecho evidente, que los problemas de salud que padecen los accionantes, sean consecuencia natural y directa de la contaminación a que aluden (Corte Constitucional, Sentencia T-1062, 2001) (Negrillas fuera de texto original).

Al final del caso la Corte Constitucional otorga la protección a los tutelantes, pero con argumentos distintos a la aplicación del 
principio de precaución. De igual manera, se analiza como los jueces de instancia desconocen la razón de ser de este principio.

Según la Corte Constitucional, existen 5 prerrogativas para aplicar el principio de precaución: 1) La existencia de peligro de daño; 2) que este sea grave e irreversible; 3) que exista un principio de certeza científica así no sea absoluta; 4) que la decisión que se tome sea para proteger el medio ambiente, y 5) que esa decisión esté motivada.

En el caso analizado, se encuentran presentes todas las prerrogativas de aplicación. El peligro de daño, manifestado en la afectación a la salud de la señora Baena, la gravedad de la afectación y la irresistibilidad a ese daño porque no puede evitarlo, la afectación al medio ambiente del conjunto residencial en el cual vive, la falta de certeza absoluta del perjuicio de las radiaciones electromagnéticas para el ser humano, y finalmente la motivación del acto en la sentencia.

Por consiguiente, se determina, la procedencia de la aplicación del principio de precaución, y se ordena el traslado de las antenas de telecomunicaciones del edificio a otro lugar lejos de este sitio residencial.

A pesar de la falta de estipulación expresa del principio de precaución como herramienta para solucionar la tutela, el caso tiene todos los requisitos de aplicación posibles y fue solucionado a favor de los tutelantes.

\section{Segundo caso (Sentencia T-299 de 2008)}

Los accionantes actuando en su nombre propio y en representación de sus hijos menores de edad, presentaron acción en contra de la Empresa de Servicios Públicos Codensa S.A., debido a la cercanía de su residencia a una subestación eléctrica, lo que podría significar la exposición y afectar la salud y el medio ambiente.

A pesar de la superación del hecho, por el retiro de la subestación eléctrica de parte de la entidad accionada, la Corte hizo alusión al principio de precaución, concluyendo el abordaje de este postulado y protección de los derechos al medio ambiente y a la salubridad teniendo en cuenta: (i) Ante la amenaza de un peligro grave al medio ambiente o la salud, del cual (ii) ausencia de certeza científica, pero 
(iii) sí existe algún principio de certeza, (iv) la toma de decisiones que propendan por la protección (Corte Constitucional, Sentencia T-299, 2008).

En la Sentencia T-299 (2008), la Corte Constitucional para el año 2008 realiza un estudio jurisprudencial respecto al principio. El magistrado ponente Jaime Córdoba Triviño ya afirmaba la constitucionalización del mismo, citando reiteradamente diversas sentencias de la misma Corporación. Se resalta que, además de ser un principio orientador de políticas, el alto Tribunal lo considera un criterio hermenéutico.

Con posterioridad la Corte Constitucional, señaló que existe afectación a humanos de los Campos ElectroMagnéticos (CEM), esos mismos emitidos por la subestación eléctrica en la propiedad horizontal. Según aquella, la Organización Mundial de la Salud (OMS) nunca ha encontrado efectos a la salud producidos por los CEM en adultos, pero en menores de edad como los hijos de los tutelantes, según estudios e investigaciones realizadas por la entidad internacional:

Mantienen la calificación de los CEM como posible carcinogénico en relación con la leucemia infantil. Esta clasificación, sin embargo, indica que los estudios científicos presentan poca evidencia en humanos sobre la relación de causalidad, de manera que debe considerarse que existe una evidencia científica limitada de un peligro potencialmente grave (Corte Constitucional, Sentencia T-299, 2008).

No se logró probar en dicha sentencia, la relación de causalidad entre la afectación a la salud y el CEM producido por la subestación, pero la Corte Constitucional con relación al desarrollo del principio de precaución y al ver arriesgada la salud de menores de edad, decidió revocar los fallos anteriores y en su lugar proteger el derecho a la integridad física en conexidad con la vida. La posibilidad de que un CEM cause una enfermedad actualmente tratable pero en la mayoría de los casos sin cura definitiva como lo es el cáncer, demuestra la actuación del principio de precaución para proteger derechos fundamentales, aun así, si están en conexidad con otros. 
$\mathrm{Al}$ estudiar este caso, es prudente considerar como acertada la decisión judicial. Existe un peligro de daño manifestado en la posible afectación a la integridad personal de los menores de edad, dado el potencial perjuicio de las ondas emitidas por la subestación. Este peligro es grave debido a la edad de los menores, e irresistible al mismo tiempo porque nada podrían hacer para evitarlo. La ciencia afirma la viabilidad de una cancerígena originada en esas ondas, pero no con seguridad absoluta.

En este caso, la medida aplicada protege el medio ambiente no solo de los menores, sino de todos los residentes cercanos a la subestación y ese actuar está motivado en la sentencia.

\section{Tercer caso. (Sentencia T-360 de 2010)}

En este caso, la demandante indicó que sufre una padecimiento coronario, por el que fue intervenida quirúrgicamente y se le instaló un cardiodesfibrilador, el cual presentó fallas debido a la instalación de una torre de telecomunicaciones de propiedad de Comcel S.A.

En primera instancia, el juez Sexto Civil Municipal de Neiva aplicó el principio de precaución en este tema, y afirmó:

Debe tenerse en cuenta, para el presente caso, el principio de precaución, debido a que se trata de "un enfoque excepcional y alternativo frente al principio de certeza científica". De igual forma, "el peligro que genera la antena para estaciones base y repetidores de red para la telefonía celular ubicada en el mismo barrio donde vive la tutelante es alto ya que las radiaciones que genera deteriora el perfecto funcionamiento del marca paso o dispositivo que le fue implantado a la tutelante precisamente para mejorar su estado de salud y garantizar su vida (Corte Constitucional, Sentencia T-360, 2010).

Sin embargo, el ad quem revocó el fallo de primera instancia alegando la falta de certeza científica en el caso concreto y que documentos emanados del propio Estado y "de la Organización Mundial de la Salud, indican que las radiofrecuencias emitidas por las antenas de la telefonía celular no son nocivas para la salud humana" (Corte Constitucional, Sentencia T-360, 2010). 
El Tribunal Constitucional en su parte motiva al revisar este fallo de tutela, se dedicó no a establecer si con esa antena se afectaba el derecho a la vida de la tutelante, sino a determinar si su marcapasos fallaba por un nexo de causalidad con la antena de Comcel. Decidió confirmar la sentencia del ad quem, exponiendo las pruebas de la situación, y adujo: "Con base en las recomendaciones y estudios científicos anteriormente citados, la Sala Sexta de Revisión no encuentra comprobado que las ondas electromagnéticas emitidas por la torre de telefonía móvil instalada en el barrio Campo Núñez de Neiva, sean las causantes del mal funcionamiento del cardio desfibrilador" (Corte Constitucional, Sentencia T-360, 2010).

En consecuencia, hizo un llamado al Ministerio de Tecnologías de la Información y las Comunicaciones y a la Comisión de Regulación de Comunicaciones para que:

(...) (ii) Igualmente, en aplicación del principio de precaución, diseñen un proyecto encaminado a establecer una distancia prudente entre las torres de telefonía móvil y las instituciones educacionales, hospitales, hogares geriátricos y centros similares, debido a que los estudios científicos analizados revelan que los ancianos y los niños pueden presentar mayor sensibilidad a la radiación de ondas electromagnéticas, estando los últimos en un posible riesgo levemente más alto de sufrir leucemia (Corte Constitucional, Sentencia T-360, 2010) (Negrillas fuera del texto original).

Si bien, no se puede concluir el nexo de causalidad entre el mal funcionamiento del cardiodesfibrilador, y las ondas emitidas por la antena de telecomunicaciones, pero es exactamente en esas situaciones donde se aplica el principio de precaución, tal cual como lo había propuesto el a quo. La Corte se esmeró en determinar si existía un nexo de causalidad, sin darse cuenta que debía ver más allá, y tutelar el derecho de la adulta mayor sin tener la absoluta evidencia de ese nexo.

Resulta contraproducente pues la alta Corporación, dispuso la existencia de una necesidad consistente en: 
Que se evalúen las medidas indicadas en la comunidad internacional, puesto que, aunque las investigaciones y estudios científicos realizados hasta la fecha no arrojen certeza de que las ondas de radiofrecuencia generadas por las estaciones base de telefonía móvil generen efectos negativos a largo plazo para la salud de la población, deben aplicarse medidas de prevención y precaución para proteger a los seres humanos de los posibles efectos nocivos, sobre todo tratándose de la población más vulnerable, como los niños y los adultos mayores (Corte Constitucional, Sentencia T-360, 2010) (Negrillas fuera del texto original).

Pero, estando en la posibilidad de aplicar las medidas necesarias de precaución para proteger a los seres humanos, como la accionante de los posibles efectos nocivos de las ondas electromagnéticas de la antena, decidió exhortar al Ministerio de Minas y Energía, para realizar políticas que protejan el derecho a la vida y medio ambiente sano de la población sin importar la completa certeza científica sobre una relación causa-efecto.

No se comparte la posición de la Corte Constitucional, porque actuó pasivamente ante una situación que requería una decisión de intervención activa, en la que se materializara la suspensión de la actividad. El hecho de exhortar al Ministerio de Minas y energía y no solucionar la situación a favor de la demandante fue una gran afectación a sus derechos. Se demostró la existencia de peligro, de daño, por las fallas del marcapasos. Ese daño es grave e irresistible, debido al peligro presente para una persona con problemas cardiacos, el fallo de su marcapasos. No existe una certidumbre científica absoluta del detrimento de esas ondas al marcapasos, solamente una creencia de ello. De haberse fallado a favor de la tutelante se habría protegido el medio ambiente y el acto estaría motivado. Todas las prerrogativas para la aplicación estaban presentes, y por una omisión de éstas, decidieron no tutelar el derecho, por tanto es prudente decir que la Corte en esta sentencia no implementó adecuadamente sus prerrogativas. 


\section{Cuarto caso (Sentencia T-104 de 2012)}

La situación fáctica analizada por la Corte Constitucional, radicaba aspectos muy importantes como la habitación digna y buen estado de un Hogar Comunitario Infantil de Matanza (Santander) donde niños recibían sus estudios en pésimas condiciones.

Se estudió el derecho a la educación presente en la Constitución y demás normas concordantes, toda vez que se ubicaron siete antenas parabólicas en el hogar comunitario. El accionante consideró que si bien se logra la optimización en la señal de televisión, puede ocasionar consecuencias perjudiciales para la salud de los menores, como descenso en los niveles de melatonina, depresión, dolor de cabeza, cáncer y leucemia infantil, fundamentado todo esto en estudios de la OMS (Corte Constitucional. Sentencia T-104, 2012).

Es de anotar que en esta posible afectación, no se demostró el nexo de causalidad de las ondas emitidas por las antenas, y alguna afectación a la salud de los niños. Como se dan las situaciones para la configuración del principio, se debe prevenir el riesgo potencial, por tanto el magistrado ponente hace una breve exposición y plantea lo siguiente: "la falta de certeza científica no puede aducirse como razón para postergar la adopción de medidas eficaces para precaver la degradación del ambiente y la generación de riesgos contra la salud", así mismo, se ordenó al Ministerio de Tecnologías de la Información y las Comunicaciones (MinTIC)y a la Agencia Nacional del Espectro, analicen las recomendaciones de la OMS, respecto a los posibles efectos adversos a la salud, con la exposición a campos electromagnéticos (Corte Constitucional. Sentencia T-104, 2012).

De igual forma, ordenó a la Alcaldía de Matanza (Santander), dentro de los tres meses siguiente, realizar un proyecto para la reubicación de las siete antenas parabólicas con relación a los hogares comunitarios y otros establecimientos de atención o permanencia de menores de edad, embarazadas, personas de tercera edad, discapacidad física, por tanto se protegieron los derechos de los niños en esta tutela.

Es claro cómo el magistrado ponente de esta sentencia, se dispone a exhortar o solicitar al MinTIC que realice políticas para prevenir 
daños graves, en aplicación al principio, pero se queda sin actuar respecto a su jurisdicción en el caso concreto, dejándole al ministerio la actuación correspondiente.

Existió un posible daño por el mal estado del sitio para la educación de los niños, siendo este grave e irresistible, debido a su edad y la imposibilidad para hacer algo al respecto, por las condiciones municipales para cambiar esta situación, hay una verdad científica, pero no absoluta porque la emisión de las ondas de parte de las antenas no es una acción completamente aceptada por la comunidad científica como afectación a la salud de los humanos. De haberse actuado se habría protegido el medio ambiente de los menores, y el acto estaría motivado.

En sentencia anterior, como la Sentencia T-299 (2008), se expuso el principio de precaución como criterio para la formulación de políticas y criterio hermenéutico. Si es criterio hermenéutico, la rama judicial tiene jurisdicción en la aplicación del principio, como lógicamente se ha expuesto en las citadas sentencias de tutela. El Magistrado Ponente (MP) en este caso, como en la Sentencia T-360 (2010), prefiere no hacerlo, y a pesar de conocer el principio, y hablar de él, no lo aplica directamente. Es por ello que muy respetuosamente no se comparte la posición de la Corte Constitucional, pues no protegió el derecho de la demandante y simplemente exhortó a la rama ejecutiva a actuar, configurándose con ello una omisión.

Ahora bien, si la misma Corte Constitucional estipula al principio precautorio como criterio hermenéutico para interpretar situaciones jurídicas, no puede desconocerlo como tal. Incurrió en un yerro para este caso

\section{Quinto caso. (Sentencia T-1077 de 2012)}

En Fresno (Tolima), Luisa María Vélez Aristizabal, menor de 15 años, quien padece de un cáncer histiocitosis de células Langerhans, y siendo sometida a intervenciones quirúrgicas y quimioterapias, que le impiden contactos con radiofrecuencias, las cuales son señales equivalentes a las electromagnéticas y por tanto dañinas. No obstante a lo anterior a 41 metros de su casa existen antenas en un predio de 
propiedad de Telefónica Telecom S.A, por lo que solicita el retiro de las mismas.

En la primera instancia, la Sala Civil-Familia del Tribunal Superior de Distrito Judicial de Ibagué se negó el amparo por:

Considerar que en este caso la menor no logró probar la vulneración o amenaza de sus derechos fundamentales. En este orden de ideas, razonó que la falta de unanimidad entre la comunidad científica al definir el impacto que tienen sobre la salud humana las ondas emitidas por las antenas de telefonía, genera la improcedencia del acción. Por consiguiente, concluyó que no existe un hecho cierto, indiscutible y probado que conlleve a predicar una vulneración o puesta en peligro de los derechos que la demandante pregona conculcados (Corte Constitucional. Sentencia T-1077, 2012) (Negrillas fuera del texto original).

La Sala del Tribunal Superior, desconoció totalmente el principio de precaución. Muy acertadamente el Procurador II Judicial Ambiental y Agrario para el Tolima quien es coadyuvante en el proceso impugnó la decisión y expresó:

Conforme al principio de precaución ambiental, debieron ser amparados los derechos fundamentales de la accionante, en razón a que la falta de certeza científica respecto de los efectos nocivos de las antenas de telefonía móvil sobre la salud humana, (...) de ningún modo puede ser óbice para tutelar las garantías constitucionales conculcadas en el presente asunto, protección que se hace más imperiosa ante el particular estado de salud de la menor de edad (Corte Constitucional. Sentencia T-1077, 2012)

La Corte Suprema de Justicia, al resolver sobre esta tutela, decidió confirmar la sentencia y negar el amparo constitucional a los derechos de la menor por falta de procedencia de la acción y que no se encuentra probado los efectos negativos en la salud, producto de las estaciones celulares (Corte Constitucional. Sentencia T-1077, 2012).

Apelando nuevamente a los argumentos del A Quo, la Corte Suprema de Justicia desconoce o malinterpreta el principio de precaución. En la sala de revisión de la Corte Constitucional, procede a conceder el 
amparo a la menor. De muchos argumentos esbozados por la Corte, respecto al principio de precaución se puede rescatar lo siguiente:

En este sentido, la omisión de regulación por parte del Estado genera un peligro de daño grave e irreversible para la salud de Luisa María Vélez Aristizabal, el cual se constata con el principio de certeza científica (conforme al aparte 2.2.4.2. de esta providencia, el término principio de certeza científica, en oposición a la certeza científica absoluta, se refiere a una duda fundada, que justifica la aplicación del principio de precaución) dado por la IARC, al calificar los campos electromagnéticos como posibles cancerígenos. Por consiguiente, atendiendo a las circunstancias del caso concreto, la Sala encuentra que, si la estación base continúa situada en el predio contiguo a la vivienda de la menor, Comcel S.A., o cualquier otra empresa operadora, podrá instalar una antena poniendo en peligro su derecho fundamental a la salud.

De manera que, se comprueba: (i) que existe peligro de daño; (ii) que éste es grave e irreversible; (iii) que existe un principio de certeza científica, de que la radiación es un posible cancerígeno; (iv) que existe la necesidad de tomar una decisión encaminada a impedir la degradación del medio ambiente de la menor, y en consecuencia, de proteger su salud (Corte Constitucional. Sentencia T-1077, 2012).

Esta sentencia desarrolla de forma coherente, las prerrogativas dejadas por la Sentencia C-293 (2002). Ha sido una de las sentencias contemporáneas desarrolladas idealmente y en un orden lógico y esquemático en miras al criterio hermenéutico precautorio. Se comparte el criterio expuesto en la sentencia de la Corte, toda vez que se demostró la existencia de un posible peligro, grave e irresistible, con alguna certeza científica pero no absoluta, encaminada a proteger el medio ambiente y a través de un acto motivado, tal como lo expuso sucintamente el magistrado ponente de esta jurisprudencia.

\section{Conclusiones}

Dentro de las agendas de los Estados, se observa claramente como punto de discusión la conservación del medio ambiente, visto como instrumento eficaz para conservar la salud del ser humano y de los recursos y ecosistemas, lo cual permite una sinergia con la 
adecuación e implementación de acciones legales con el fin de buscar su protección.

El principio de precaución es destinado a la protección del medio ambiente, por el cual en presencia de una situación donde se tiene alguna razón para inferir que una acción podría causar daño al medio ambiente, a pesar de no tener la completa y absoluta certeza científica de su menoscabo a causa de dicha acción, se debe actuar para evitar esos posibles daños.

Si bien, una primera justificación se encuentra en la protección de derechos colectivos como el medio ambiente, su protección de impacto está ligada de manera inescindible con la vida del hombre, y su inclusión como miembro activo de una sociedad, elemento estructural del Estado.

Para establecer si en Colombia a nivel de acciones de tutela revisadas por la Corte Constitucional se ha llevado una configuración real y cierta del principio precautorio se analizaron diversas sentencias del órgano de cierre citado, entre los años 2001 a 2019. Luego de haber estudiado cada sentencia y verlas todas en conjunto, se logró determinar y concluir que:

El principio de Precaución o precautorio está constitucionalizado, conforme al preámbulo (Constitución Política de Colombia, 1991):

Artículo 78. La ley regulará el control de calidad de bienes y servicios ofrecidos y prestados a la comunidad, así como la información que debe suministrarse al público en su comercialización. Serán responsables, de acuerdo con la ley, quienes en la producción y en la comercialización de bienes y servicios, atenten contra la salud, la seguridad y el adecuado aprovisionamiento a consumidores y usuarios. El Estado garantizará la participación de las organizaciones de consumidores y usuarios en el estudio de las disposiciones que les conciernen. Para gozar de este derecho las organizaciones deben ser representativas y observar procedimientos democráticos internos.

Artículo 79. Todas las personas tienen derecho a gozar de un ambiente sano. La ley garantizará la participación de la comunidad en las decisiones que puedan afectarlo. Es deber del Estado proteger la diversidad e integridad del ambiente, conservar las áreas de especial importancia ecológica y fomentar la educación para el logro de estos fines. 
Artículo 80. El Estado planificará el manejo y aprovechamiento de los recursos naturales, para garantizar su desarrollo sostenible, su conservación, restauración o sustitución. Además, deberá prevenir y controlar los factores de deterioro ambiental, imponer las sanciones legales y exigir la reparación de los daños causados. Así mismo, cooperará con otras naciones en la protección de los ecosistemas situados en las zonas fronterizas.

Artículo 226. El Estado promoverá la internacionalización de las relaciones políticas, económicas, sociales y ecológicas sobre bases de equidad, reciprocidad y conveniencia nacional.

La Corte Constitucional ha determinado que la esfera de análisis del principio de precaución tiene una aplicación amplia en acciones populares para la protección de los derechos colectivos y a través de la acción de tutela puede ser amparado el principio de precaución, debido entre otras razones a su relación inescindible con los derechos fundamentales como la vida, la integridad física, la salud, los derechos de los niños. En ambas esferas como resultados de los compromisos internacionales, que exigen del juez ser garante de esos presupuestos, dentro de los elementos de una justicia ambiental constitucional e internacional.

Si bien el principio precautorio ha sido desarrollado por la jurisprudencia a partir de la Ley 99 (1993), no ha significado por sí mismo una adecuada configuración. Se analizó como varias veces la Corte ha caído en errores al momento de su interpretación. A pesar, de haber comprendido bien de qué se trata, y a dónde va encaminada la precaución, en ocasiones su hermenéutica de abordaje, ha determinado que no existen evidencias de un posible daño, a las distintas tipologías de los derechos constitucionales, con el fin de acreditar una posible afectación o daño al bien jurídico protegido, como sucede en la Sentencia T-104 (2012) y Sentencia T-360 (2010). Entiende el principio como guía para realizar políticas, pero no se percata de su carácter hermenéutico, explicado en Sentencia T-299 (2008).

A pesar del desarrollo jurisprudencial, la mayoría de los jueces de instancia, incluso altas cortes como la Corte Suprema de Justicia, no le ha dado la aplicación al principio de precaución, conforme a 
la hermenéutica constitucional que existe frente a su desarrollo en distintos casos, como por ejemplo la Sentencia T-1077 (2012) vulnera derechos fundamentales, siendo el órgano de cierre la Corte Constitucional a través de su eventual revisión para subsanar el error de interpretación.

El principio de precaución, constituye un parámetro de hermenéutica constitucional, que fundamenta la decisión en las acciones constitucionales, actos administrativos, decisiones de las autoridades ambientales; que resulta garante aplicar su esfera de aplicación con el fin de salvaguardar bienes jurídicos constitucionales e internacionales.

\section{REFERENCIAS}

Agudelo, G. y López, J. F. (2009). El principio de precaución en el plan Nacional de Salud Pública de Colombia: ¿Ausencia o un gran mal? Laguna Azul, (28), 15-23. Obtenido de http:// www.scielo.org.co/pdf/luaz/n28/n28a02.pdf

Andorno, R. (2008). Principio de precaución. En, J. Tealdi (coord.), Diccionario Latinoamericano de Bioética, vol. II (p. 345347). Bogotá, D.C.: Unibiblos y Red Latino Americana y del Caribe de Bioética de la UNESCO. Recuperado de http:// www.unesco.org.uy/shs/fileadmin/templates/shs/archivos/ DicoParteII.pdf

Bechara, A. (2018). Investigación-acción-jurídica: escenarios para una investigación activa y crítica en el Derecho. Jurídicas CUC, 14(1), 211-232. http://dx.doi.org/10.17981/juridcuc.14.1.2018.10

Cafferatta, N. A. (2009). El principio precautorio. Buenos Aires: Instituto Nacional de Ecología. Disponible en http:// ebookcentral.proquest.com/lib/bibliotecaunimagsp/detail. action?docID $=3183650$

De Cozár, J. M. (2005). El principio de precaución y medio ambiente. Salud Pública, 79(2), 133-144. Obtenido de http://scielo. isciii.es/pdf/resp/v79n2/colaboracion1.pdf 
Drnas, Z. (2009). El principio de precaución ambiental. La practica Argentina. Córdoba: Lerner. Recuperado de https://revistas.unc.edu.ar/index.php/recordip/article/view/34/24

Granja, H. (2010). Nuevos riesgos ambientales y derecho administrativo. [Tesis inédita]. Universidad del Rosario, Bogotá, D.C., Colombia.

Kamada, L. E. (2012). Del paradigma de la certeza al paradigma de la incertidumbre como criterio de decisión judicial en materia ambiental. Infojus, [DACF120104], 1-30. Recuperado de https://www.justiciajujuy.gov.ar/escuela-de-capacitacion/images/Doctrina_Local/EL_PARADIGMA_DE_ LA_PRECAUCION_-_Luis_E_Kamada.pdf

ONU. UNCED. (2012). Conferencia de las Naciones Unidas sobre el Desarrollo Sostenible (Río+20). [A/CONF.216/L.1]. Disponible en https://rio20.un.org/sites/rio20.un.org/files/aconf.216-1-1_spanish.pdf.pdf

ONU. UNFCCC. (1992). Convención marco de las Naciones Unidas sobre el cambio climático. [FCCC/INFORMAL/84]. Disponible en https://unfccc.int/resource/docs/convkp/convsp.pdf

Pabón, J. (2018). Notas acerca de la Democracia en Norberto Bobbio. Jurídicas CUC, 14(1), 9-28. https://doi.org/10.17981/ juridcuc.14.1.2018.01

Reales, A. (2016). La democracia representativa en el marco de los derechos humanos en Colombia. Jurídicas CUC, 12(1), 9-16. Recuperado de https://revistascientificas.cuc.edu.co/ juridicascuc/article/view/1112

República de Colombia. Asamblea Nacional Constituyente. (1991). [Constitución política de Colombia]. Gaceta Constitucional, 1(114).

República de Colombia. Corte Constitucional. (28 Enero de 2019). Expediente T-6.809.212. [Sentencia T-021]. MP: Alberto Rojas Ríos.

República de Colombia. Corte Constitucional. (21 de Abril de 2017). Expediente T-4.245.959. [Sentencia T-236]. MP: Aquiles Arrieta Gómez. 
República de Colombia. Corte Constitucional. (20 de Febrero de 2012). Expediente T-3228384. [Sentencia T-104]. MP: Nilsón Pinilla Pinilla.

República de Colombia. Corte Constitucional. (12 de Diciembre de 2012). Expediente T-3.286.371. [Sentencia T-1077]. MP: Jorge Ignacio Pretelt Chaljub.

República de Colombia. Corte Constitucional. (11 de Mayo de 2010). Expediente T-2520375. [Sentencia T-360]. MP: Nilsón Pinilla Pinilla.

República de Colombia. Corte Constitucional. (27 de julio de 2010). Expediente D-7977. [Sentencia C-595]. MP: Jorge Iván Palacio Palacio.

República de Colombia. Corte Constitucional. (6 de Septiembre de 2010). Expediente D-8019. [Sentencia C-703]. MP: Gabriel Eduardo Mendoza Martelo.

República de Colombia. Corte Constitucional. (2 de Abril de 2008). Expediente T-1.759.107. [Sentencia T-299]. MP: Jaime Córdoba Triviño.

República de Colombia. Corte Constitucional. (23 de Abril de 2002). Expediente D-3748. [Sentencia C-293]. MP: Alfredo Beltrán Sierra.

República de Colombia. Corte Constitucional. (28 de Junio de 2001). Expediente LAT-191. [Sentencia C-671]. MP: Jaime Araujo Rentería.

República de Colombia. Corte Constitucional. (11 de Octubre de 2001). Expediente T-470.483. [Sentencia T-1062]. MP: Álvaro Tafur Galvis.

República de Colombia. Congreso de Colombia. (24 de abril de 2012). Por la cual se adopta la política nacional de gestión del riesgo de desastres y se establece el Sistema Nacional de Gestión del Riesgo de Desastres y se dictan otras disposiciones. [Ley 1523]. DO: 48.411.

República de Colombia. Congreso de Colombia. (28 de octubre de 1994). Por medio de la cual se aprueba la "Convención Marco de las Naciones Unidas sobre el Cambio Climático”, hecha en Nueva York el 9 de mayo de 1992. [Ley 164]. DO: 41.575. 
República de Colombia. Congreso de Colombia. (22 de diciembre de 1993). Por la cual se crea el Ministerio del Medio Ambiente, se reordena el Sector Público encargado de la gestión y conservación del medio ambiente y los recursos naturales renovables, se organiza el Sistema Nacional Ambiental, SINA, y se dictan otras disposiciones. [Ley 99]. DO: 41.146. República de Colombia. Presidencia de la República. (19 de noviembre de 1991). Por el cual se reglamenta la acción de tutela consagrada en el artículo 86 de la Constitución Política de Colombia. [Decreto 2591]. DO: 40.165.

Rodríguez, G. y Vargas, I. (2017). Principio de precaución: Desafíos $y$ escenarios de debate. Bogotá, D.C: Temis. Recuperado de http://ebookcentral.proquest.com/lib/bibliotecaunimagsp/ detail.action?docID $=5308354$

Sánchez, E. (2004). El principio de precaución: Implicaciones para la salud pública. Gaceta sanitaria. Sociedad Española de Salud Pública y Administración Sanitaria, 16(5), 371-373. https://doi.org/10.1016/S0213-9111(02)71944-4

Doctora en Derecho de la Universidad Santo Tomas (Colombia). Magister en Derecho de la Universidad Sergio Arboleda (Colombia). Especialista en Derecho Probatorio y especialista en Derecho Comercial y Marítimo de la Universidad Sergio Arboleda. Abogada egresada de la Universidad Sergio Arboleda. Perteneciente al Grupo de Investigación de la Facultad de Humanidades del Programa de Derecho de la Universidad del Magdalena (Santa Marta, Colombia) Saberes Jurídicos Grisjum en la línea de investigación: Naturaleza, cultura y sociedades. https://orcid.org/0000-0002-6424-8596 